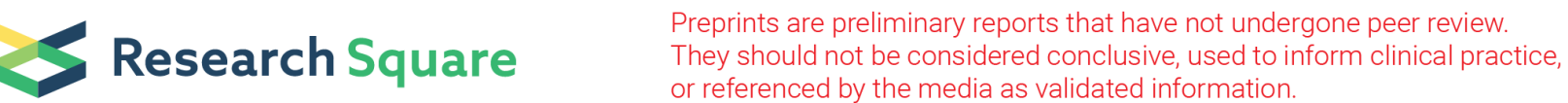

\section{More Conservative Rehabilitation Strategy within Postoperative 6 Month for All-Inside Reconstruction Compared with Anatomical Reconstruction of Anterior Cruciate Ligament may be able to Achieve Better Stability: Based on the Study of Graft Maturity}

Rubing Lin

Peking University Shenzhen Hospital, Clinical College of Anhui Medical University

Qiuwen Zhong

Peking University Shenzhen Hospital

Xiao Wu

Peking University Shenzhen Hospital

Lei Cui

Peking University Shenzhen Hospital, Clinical College of Anhui Medical University

Rong Huang

Peking University Shenzhen Hospital

Qianhua Deng

Peking University Shenzhen Hospital

Changqing Jiang

Huazhong University of Science and Technology Union Shenzhen Hospital

Wei Li (D Iwjnu2003@126.com)

Peking University Shenzhen Hospital

\section{Research Article}

Keywords: All-inside, Single-bundle, Anterior cruciate ligament reconstruction, Graft maturity, Stability

Posted Date: November 11th, 2021

DOI: https://doi.org/10.21203/rs.3.rs-989313/v1

License: (c) (i) This work is licensed under a Creative Commons Attribution 4.0 International License.

Read Full License 


\section{Abstract}

Background: Individualized rehabilitation based on graft maturity is necessary to optimize patient function and prevent graft re-injury. But there is a lack of studies on graft maturity in the all-inside singlebundle anterior cruciate ligament reconstruction.

Hypothesis/Purpose: Compared to the difference in graft maturity, functional scores, and stability between all-inside and anatomical single-bundle anterior cruciate ligament reconstruction, it was assumed that the stability of all-inside reconstruction is relatively insufficient, and its graft maturity is worse than the anatomical reconstruction.

Study Design: Randomized controlled clinical trial.

Methods: Fifty-four patients were recruited in this study and randomly assigned to the all-inside reconstruction group $(n=27)$ and the anatomical reconstruction group $(n=27)$ using the same rehabilitation strategy. The Tegner, International Knee Documentation Committee, and Lysholm score were recorded at postoperative $3^{\text {rd }}, 6^{\text {th }}$, and $12^{\text {th }}$ month to assess functional recovery. Magnetic resonance imaging was conducted to measure the Signal/Noise quotient (SNQ) of the intra-articular graft to observe the maturity. Stability was assessed using GNRB relaxation measuring instrument at the postoperative $12^{\text {th }}$ month.

Results: The graft SNQ of the all-inside group was significantly higher than that in the anatomical group during the postoperative $6^{\text {th }}$ month $(p<0.05)$. There was no statistical difference in graft SNQ between the two groups at postoperative $3^{\text {rd }}$ and $12^{\text {th }}$ month $(p>0.05)$. Both groups exhibited the graft's highest SNQ in the middle region, followed by the proximal region, and the lowest was the distal region. Functional scores improved significantly in both groups and had no statistical difference $(p>0.05)$. The stability recovered well in both groups during the postoperative $12^{\text {th }}$ month, but the GNRB relaxation was higher in the all-inside group $(p<0.05)$. There was no correlation between functional scores and graft maturity in the two groups $(p>0.05)$.

Conclusions: All-inside reconstruction graft maturity is inferior to the anatomical reconstruction during postoperative $6^{\text {th }}$ month, which may cause relatively insufficient stability of all-inside reconstruction compared with anatomical reconstruction under the same rehabilitation strategy. A more conservative rehabilitation strategy for all-inside reconstruction around the postoperative $6^{\text {th }}$ month may achieve better stability.

\section{Introduction}

An anterior cruciate ligament (ACL) tear is one of the most common sports-related injuries [1, 2]. The lack of active treatment often causes joint instability, hence, increasing the risk of osteoarthritis [3]. From the first attempt to repair ACL by Mayo and Robson [4] in 1895, to the anatomical single-bundle ACL reconstruction became mainstream gradually in 1995, then to the first generation of all-inside single- 
bundle ACL reconstruction technique (AIT-ACLR) by Lubowitz [5] in 2006, and to the second generation refinement in 2011 [6]. At this time, AIT-ACLR has been greatly simplified and attracted attention. Lubowitz proposed that the "bone socket" structure used in AIT-ACLR could reduce the accumulation of bioinvasive factors, such as joint fluid and thus slow down the widening of the tunnel [7]. Many studies have also concluded that there is no difference between AIT-ACLR and anatomical single-bundle ACL reconstruction in functional recovery. Still, AIT-ACLR is better than the latter in pain reduction and slowing tunnel widening [8-12]. However, some authors have concluded that knee stability after AIT-ACLR is insufficient $[13,14]$ and has a higher graft failure rate than anatomical reconstruction [15]. The reason for the inadequate stability and high refurbishment rate after AIT-ACLR is still highly controversial, which may be related to the premature return to motion when the graft is immature or at least not fully healed. Unfortunately, the research on AIT-ACLR graft maturity is rare, leading to the inability to develop a rehabilitation strategy that conforms to graft maturity law.

Ma et al [16] concluded that individualized rehabilitation based on graft maturity is necessary to optimize patient function and prevent graft re-injury. Van et al [17] also concluded that magnetic resonance imaging (MRI)-based assessment of graft maturity is essential for selecting the appropriate time to resume exercise after surgery. Therefore, the study of graft maturity after AIT-ACLR will help make reasonable and accurate rehabilitation decisions. In this study, it was assumed that the stability of allinside reconstruction is relatively insufficient, and its graft maturity is worse than anatomical reconstruction. The difference in graft maturity, functional scores, and stability between all-inside and anatomical single-bundle ACL reconstruction will be compared, which will help make better rehabilitation strategy.

\section{Method}

This was a prospective randomized controlled trial approved by the hospital's ethics review committee and registered at the national clinical registration center. Clinical Protocol for the clinical trial is included as a Supplement.

\section{Participants}

Fifty-four patients who underwent ACL single-bundle reconstruction in our department between September 2018 to July 2019 were enrolled. This54 patients were randomly assigned to an AIT-ACLR group (all-inside group, $n=27$ ) and the anatomical single-bundle ACL reconstruction group (anatomical group, $n=27$ ) using the random number table method. The inclusion criteria for participants were (1) history of acute trauma, and MRI showing ACL discontinuity or hyperintense signal, (2) age 18-45 years, (3) $18 \leq$ body mass index (BMI) $\leq 28$, and (4) patient's informed consent and signed relevant documents. The participants were excluded if they had any of the following: (1) injury-time $>12$ months, (2) combined with remaining ligament injury, (3) history of ACL reconstruction surgery, (4) patients with ligamentous release signs, (5) patients with severe underlying disease or uncooperative follow-up, and (6) bilateral ACL injury. 


\section{Clinical Evaluation}

The Tegner, IKDC, and Lysholm scores were recorded during postoperative 3rd, 6th, and 12th months to assess functional recovery. During postoperative 12th month, GNRB (Genourob, France) relaxation measuring instrument was performed to record the tibial anterior movement gap between knees under 134-N load and to assess stability. Data collectors and patients were not aware of the surgical approach. Only the surgeon was aware of the grouping. The study procedure is indicated in Figure 1.

\section{MRI Analysis}

The Signal/Noise quotient (SNQ) of the proximal, middle and distal regions of interest (ROI) of the intraarticular graft was measured using 3.0 T MRI during postoperative 3rd, 6th, and 12th months to observe the graft maturity law. From a sagittal T-two image, to select the interface that clearly showed graft and quadriceps tendon ROI. Graft ROI was located at the "proximal (near the femoral tunnel)," "distal (near the tibial tunnel)," and the "middle (between them)." Quadriceps tendon ROI was located at 2-cm above the patellar attachment point. The signal value of the 2-cm anterior to the tibial tuberosity was used as the background signal. ROI signal measurement area was controlled at 10-sq.mm (with an allowable error of 0.5-sq.mm or less). Graft SNQ = (Graft ROI mean signal value - Quadriceps tendon ROI mean signal value)/Background mean signal value. See Figure 2 for details.

\section{Surgical Techniques}

The same group of experienced surgeons performed all procedures. The patient was placed in the supine position after lumbar anesthesia, with the lower leg hanging down over the edge of the bed. A subpatellar anteromedial and anterolateral approach was taken to place the knee arthroscopy, and a planing knife was used to clean the subpatellar fat pad and synovial membrane, exploring the injury, the ACL tibial and femoral stops were cleaned, and the posterior edge of the lateral wall of the intercondylar socket of the femur was exposed. An anteromedial approach was added, and the entry point was prepped using a microfracture cone and marked slightly posterior above the midpoint of the lateral wall of the resident's crest.

The all-inside reconstruction technique was performed using an anteromedial approach, by an inverted drill (Arthrex, USA) to drill the femoral bone tract 2-vm in length in a retrograde fashion (see Figure 3 for details). The anatomical reconstruction technique was also performed using an anteromedial approach, using an ordinary drill to drill the bone tract in a prograde fashion, with the size of the bone tract depending on the diameter of the grafted tendon. For tibial tunnel creation, both all-inside reconstruction and the anatomical reconstruction had an internal port located at the center of the C-shaped stop of the ACL tibia and an external port located approximately 3-cm medial to the tibial tuberosity. For the all-inside reconstruction, the tibial tunnel was also drilled using an inverted drill (Arthrex, USA), and the drill wing was opened to enlarge the tract retrogradely to a depth of approximately $2-\mathrm{cm}$. For the anatomical 
reconstruction, a guide pin was placed first, and the full-length tibial tunnel was drilled thoroughly along the guide pin.

To harvest the autologous hamstring tendon, both techniques were performed with an oblique incision of the skin at approximately $3-\mathrm{cm}$ medial to the tibial tuberosity to expose the semitendinosus and gracilis tendon, and the tendon was retrieved using a tendon retriever. All-inside reconstruction needed only a single harvest of the semitendinosus tendon, and the removed semitendinosus tendon was double-folded to make a four-stranded single-bundle structure (see Figure 3 for details) The ends of the graft tendon were connected with an adjustable steel plate using a rope loop. The diameter and length of the graft were recorded intraoperatively. The prepared grafted tendon was entered through the arthroscopic port, and the retraction cord guided the grafted tendon through and filled the femoral tunnel, and then the adjustable steel plate was stuck outside the bone cortex and tightened and knotted to fix it. Next, the tibial guide cord was tractored to the tibial tunnel along with the lead on the other side of the grafted tendon, and the traction tibial guide cord pulled the tendon lead out pulling the tendon into the tibial tunnel. Anatomical reconstruction requires to harvest the semitendinosus and gracilis tendon, folding them in half to prepare a four-stranded single-bundle structure, and attaching an adjustable steel plate to one end of the graft tendon, and securing it with an ordinary traction wire at the other end. The graft end with an adjustable steel plate was guided through the tibial tunnel to the femoral tunnel, and then the steel plate was stuck outside the femoral cortex and fixed with a knot, and another graft end on the tibial side was fixed with absorbable compression screws.

After complete fixation of the two types of grafts, arthroscopic confirmation of the tendon without torsion and good tension was performed, and the tension was adjusted to determine whether it was isometric and whether there was intercondylar impingement between the graft and femur. The joint cavity was flushed, and the incisions were sutured and bandaged using pressure after reexamination, and the affected limb was fixed with a straight leg brace and returned to the ward.

\section{Postoperative Rehabilitation}

The same rehabilitation protocol was used in both groups. The patient was gradually weight-bearing on the affected limb from the 3rd day to the 1st week after surgery. From 2nd to the 4th week, the patient practiced knee flexion with a goal of 90 degrees. During postoperative 4 th week, the crutches were abandoned, and full weight-bearing was achieved. During postoperative $3 \mathrm{rd}$ month, the knee was flexed to 130 degrees, and squatting exercises against the wall were started-flexibility training and technique training during postoperative 4th month. Return to non-contact sports activities at 6 th month after surgery.

\section{Statistical Analysis}

IBM SPSS 21.0 statistical software was applied to data analysis. The measurement data were expressed as mean \pm standard deviation $(\mathrm{M} \pm \mathrm{SD}$ ). For comparison between two independent groups, the data met the normal distribution using independent samples t-test and Pearson correlation analysis. The data did 
not meet the normal distribution using Mann-Whitney U-test and Spearman correlation analysis. $p<0.05$ is considered statistically significant.

\section{Results}

\section{Patient Demographics}

Fifty-four patients were enrolled in the study between Sep 2018 and Jul 2019 and were randomly assigned to the all-inside group $(n=27)$ and the anatomical group $(n=27)$. During the follow-up, one case lost follow-up in the anatomical group and two cases in the all-inside group because of the patient's workplace. There were no statistical differences in demographic data between the two groups $(p>0.05)$. The graft length was significantly shorter in the all-inside group than the anatomical group $(p=0.000)$. There was no statistically significant difference in graft diameter between the two groups $(p>0.05)$. See Table 1 for details.

Table 1

Demographic Data and Graft size of the Participants

\begin{tabular}{|llll|}
\hline Demographic Data & $\begin{array}{l}\text { All-inside group }(\mathbf{n}= \\
\mathbf{2 5})\end{array}$ & $\begin{array}{l}\text { Anatomical group }(\mathbf{n}= \\
\mathbf{2 6})\end{array}$ & P value \\
\hline Men: women, $\mathrm{n}$ & $22: 3$ & $23: 3$ & $\mathrm{n} . \mathrm{s}$. \\
\hline Operative side, left/right, $\mathrm{n}$ & $9 / 16$ & $12 / 14$ & $\mathrm{n} . \mathrm{s}$. \\
\hline Age, mean $\pm \mathrm{SD}, \mathrm{y}$ & $31.3 \pm 5.8$ & $29.9 \pm 4.6$ & $\mathrm{n} . \mathrm{s}$. \\
\hline $\begin{array}{l}\text { Body mass index, mean } \pm \mathrm{SD}, \\
\mathrm{kg} / \mathrm{m}^{2}\end{array}$ & $24.0 \pm 3.2$ & $23.6 \pm 2.5$ & $\mathrm{n} . \mathrm{s}$. \\
\hline \begin{tabular}{l} 
Diameter of grafts, mean $\pm \mathrm{SD}, \mathrm{mm}$ \\
\hline Length of grafts, mean $\pm \mathrm{SD}, \mathrm{cm}$
\end{tabular} & $7.9 \pm 0.3$ & $7.8 \pm 0.3$ & $\mathrm{n} . \mathrm{s}$. \\
\hline n.s. No significant difference between the two groups, $p>0.05$. & & $0.000 *$ \\
\hline *Statistical significance between the two groups, $p<0.05$. & & \\
\hline
\end{tabular}

\section{Clinical Findings}

Before the operation and during postoperative 3rd, 6th, 12th month, there was no statistical difference in Lysholm, Tegner, and IKDC scores between the all-inside and anatomical group $(p>0.05)$. Stability was well restored in both groups during postoperative 12th month, but GNRB relaxation was greater in the allinside group than in the anatomical group, with a statistical difference $(p=0.048<0.05)$. See Table 2 for details. 
Table 2

Clinical Outcomes in the All-inside and Anatomical Group

\begin{tabular}{|lllll|}
\hline Groups & Preoperative & 3 month & 6 month $^{\#}$ & 12 month $^{\#}$ \\
\hline All-inside group & & & & \\
\hline Tegner score & $2.70 \pm 1.84$ & $2.70 \pm 0.95$ & $4.15 \pm 1.13$ & $6.70 \pm 0.95$ \\
\hline IKDC score & $50.98 \pm 22.03$ & $60.88 \pm 14.52$ & $73.96 \pm 11.95$ & $87.37 \pm 6.72$ \\
\hline Lysholm score & $58.35 \pm 22.18$ & $76.52 \pm 14.40$ & $86.41 \pm 9.41$ & $94.19 \pm 6.16$ \\
\hline GNRB & $\mathrm{N}$ & $\mathrm{N}$ & $\mathrm{N}$ & $2.87 \pm 1.23^{\star}$ \\
\hline Anatomicalal group & & & & \\
\hline Tegner score & $2.78 \pm 1.85$ & $2.59 \pm 0.84$ & $4.44 \pm 1.01$ & $6.52 \pm 1.05$ \\
\hline IKDC score & $47.04 \pm 22.53$ & $59.74 \pm 13.82$ & $78.34 \pm 9.20$ & $86.86 \pm 8.18$ \\
\hline Lysholm score & $55.00 \pm 22.63$ & $76.74 \pm 12.53$ & $87.81 \pm 6.93$ & $92.67 \pm 5.76$ \\
\hline GNRB & $\mathrm{N}$ & $\mathrm{N}$ & $\mathrm{N}$ & $2.27 \pm 1.34^{\star}$ \\
\hline
\end{tabular}

\#Clinical outcomes in the all-inside and anatomical group before operation and then at postoperative 3rd, 6th, and 12th month. Values are shown as mean \pm SD. N: Not measured.

${ }^{*}$ Comparison between the two groups have a significant difference, $p \otimes 0.05$.

\section{MRI Graft Maturity Findings}

There were no cases of revision surgery in either group, and there were no adverse effects such as infection or dysfunction. The MRI was performed during postoperative 3rd, 6th, and 12th months. See Figure 4 for details.

The graft SNQ in the all-inside group tended to increase from postoperative 3rd month to 6th month and gradually decreased from postoperative 6th month to 12th month. The graft SNQ in the anatomical group gradually increased with time during the follow-up period. The graft SNQ law of the all-inside group presented a "mountain" shape and the anatomical group graft SNQ law presented a "staircase" shape (see Figure 5 for details). Both groups showed the highest SNQ in the middle ROI of the graft, followed by the proximal region, and the lowest was the distal region.

There was no statistical difference in the graft SNQ between the two groups during postoperative $3^{\text {rd, }}$ and 12th months $(p>0.05)$. At the postoperative 6th month, the graft SNQ increased in both groups than the postoperative 3rd month. Still, the increase in the all-inside group was more obvious and reached a peak, and there was a statistical difference between the two groups $(p<0.05)$. See Figure 6 for details.

\section{Correlation Analysis}


Correlation analysis between functional scores and the SNQ of the proximal, distal, and middle ROI of the all-inside and anatomical group at postoperative 3rd, 6th and 12th months. There was no correlation between functional score and graft maturity for both groups $(p>0.05)$. See Table 3 for details.

Table 3

Correlation Analysis Between Functional Score and Graft SNQ \#

\begin{tabular}{|c|c|c|c|c|c|c|c|}
\hline \multirow[t]{2}{*}{$P$ value* } & & \multicolumn{3}{|c|}{ All-inside group $(n=25)$} & \multicolumn{3}{|c|}{ Anatomical group $(n=26)$} \\
\hline & & Proximal & Middle & Distal & Proximal & Middle & Dista \\
\hline \multirow[t]{3}{*}{ Tegner } & 3 month & 0.671 & 0.641 & 0.523 & 0.429 & 0.472 & 0.975 \\
\hline & $6 \mathrm{~m}$ & 0.677 & 0.738 & 0.348 & 0.244 & 0.931 & 0.826 \\
\hline & $12 \mathrm{~m}$ & 0.218 & 0.243 & 0.341 & 0.980 & 0.928 & 0.908 \\
\hline \multirow[t]{3}{*}{ IKDC } & 3 month & 0.463 & 0.938 & 0.542 & 0.101 & 0.154 & 0.986 \\
\hline & $6 \mathrm{~m}$ & 0.729 & 0.659 & 0.818 & 0.672 & 0.110 & 0.796 \\
\hline & $12 \mathrm{~m}$ & 0.810 & 0.422 & 0.583 & 0.395 & 0.424 & 0.966 \\
\hline \multirow[t]{3}{*}{ Lysholm } & 3 month & 0.859 & 0.364 & 0.702 & 0.174 & 0.269 & 0.796 \\
\hline & $6 \mathrm{~m}$ & 0.952 & 0.627 & 0.930 & 0.190 & 0.667 & 0.740 \\
\hline & $12 \mathrm{~m}$ & 0.395 & 0.156 & 0.810 & 0.097 & 0.163 & 0.062 \\
\hline
\end{tabular}

\# Correlation analysis between functional scores and the SNQ of the proximal, distal, and middle ROI of the all-inside and anatomical group during postoperative $3 \mathrm{rd}, 6^{\text {th, }}$ and 12 th month.

*All P values were greater than 0.05 ; there was an insignificant correlation.

\section{Discussion}

ACL rupture is now mostly advocated to be reconstructed arthroscopically using autologous or allograft tendons. After reconstruction, the graft underwent a biological healing process to become a mature graft [18]. Graft healing consists of two regions of healing: intra-bone tunnel healing and intra-articular healing, in which there are three distinct stages of the intra-articular healing process of the graft, namely, early cellular necrosis, cellular regeneration and hematologic reconstruction, and graft remodeling $[19,20]$. Early necrosis occurs within four weeks postoperatively and is characterized by graft necrosis and an abnormal decrease in cell numbers [21,22]. Cellular regeneration and hematologic reconstitution begin at postoperative 4th -12th week [23] when the greatest changes in cellular activity and extracellular matrix occur. A certain degree of rehabilitation load is helpful to stimulate cellular regeneration and hematologic reconstitution. Still, due to the knowledge regarding mechanism underlying of the graft are limit at this stage, and excessive load should be avoided to damage the graft [21]. The remodeling phase begins 
during the postoperative 12th week, and this process is a continuous transformation of graft morphology and mechanical strength to normal ACL.

Many factors influence the biological healing process of these grafts. The commonly known factors include the preservation of the ACL stump, preservation of the popliteal tendon stop, or the location of the bone tunnel-opening pattern, the type of graft, and bending angle. Some studies have concluded that the retention of the stump has a positive effect on graft synovialization and thus promoting graft maturity [24]. However, it has been suggested that stump can increase the intra-articular volume causing intercondylar socket impingement, which causes an increased incidence of postoperative limited knee extension [25], so whether to preserve the stump for ACL reconstruction is controversial and requires further clinical validation. Liu et al [26] found that graft MRI signal intensity was relatively low and unchanged after ACL reconstruction with preservation of the popliteal stop, and Ruffilli et al [27] also concluded that grafts with stop preservation, had good morphology and better maturity. This may be because the retention of the stop can somewhat bypass the first two stages of biological healing and thus enhance the ligamentization process, but there are still relatively few studies, and the long-term efficacy needs to be demonstrated further. Graft bending angle can also influence early maturity [28], where the anatomical positioning of the femoral tunnel is an essential factor influencing the graft bending-angle.

In fact, the tunnel structure, graft fixation, and rehabilitation strategies may also have a critical combined effect on graft maturity. This study showed that the maturity of the middle region of the intra-articular graft after all-inside and anatomical reconstruction performed the worst, followed by the distal region. It may since the middle region of the graft is the main part subjected to distraction forces, and the distal region of the graft is close to the tibial tunnel that easily affected by the tibial tunnel. Because the tibial tunnel is the main difference between all-inside and anatomical reconstruction, and some studies believe that all-inside reconstruction cortical suspension fixation is prone to produce mechanical effects such as "bungee" and "wiper" [29] in the early stage, while the anatomical reconstruction with interference screw fixation is relatively firmer in the early stage, which may be the reason why all-inside reconstruction graft maturity is worse than anatomical reconstruction in the first six months after surgery. However, the unique "bone socket" structure used in all-inside reconstruction may be the major reason for the trend of getting worse first then getting better of graft maturity. Because the blind end of the "bone socket" structure is a good barrier to the bone destruction by bioerosive factors such as joint fluid and promotes tendon-bone healing, increases the stability of graft attachment in the tunnel, inhibits the mechanical effects of pulling and friction, thus, accelerating graft maturity.

It is worth noting that even though graft maturity after all-inside reconstruction improved well in the late follow-up period, it was found that knee stability after all-inside reconstruction was not better than that of anatomical reconstruction, which may be related to returning to exercise prematurely when the graft was immature after all-inside reconstruction. Suppose the difference in graft maturity between the two techniques is ignored, and the same rehabilitation intensity is used, the graft of all-inside reconstruction cannot withstand the load and anatomical reconstruction, which will cause laxity, severe ones leads to 
dislocation and fracture, which will eventually require revision surgery. Darren et al[30] mentioned in a systematic evaluation that $61.5 \%$ of the studies on all-inside techniques allowed jogging at $2-4$ months postoperatively, and $69.2 \%$ allowed cutting and rotational movements to resume at 6-9 months postoperatively. The fact that so many studies chose to allow patients to return to exercise up to six months postoperatively may be because they made rehabilitation decisions based solely on postoperative functional recovery and ignored the role of graft maturity. This study concluded that good improvement in knee function was obtained after all-inside reconstruction, but there was no correlation between functional recovery and graft maturity. Good functional recovery did not mean that the graft was well matured, which is consistent with the studies of Marcus and Zhang et al [31, 32], so it is unreasonable to make rehabilitation decisions based on postoperative functional recovery alone, and we still need to consider the graft maturity.

In clinical practice, the appropriate time to restore exercise status after all-inside reconstruction is often based on the rehabilitation strategy of anatomical reconstruction. Sill, this study shows that there is a significant difference in graft maturity between all-inside reconstruction and anatomical reconstruction during postoperative 6th month, only after that there is a gradual improvement, and Insignificant difference in graft maturity between the two groups at one year after surgery. Therefore, it is necessary to adopt a more conservative rehabilitation strategy after all-inside reconstruction than anatomical reconstruction, mainly to avoid premature and overly aggressive rehabilitation training around postoperative 6th month. Follow-up, further research is required to develop scientific rehabilitation strategies that match the graft maturity of the all-inside reconstruction so that a better recovery of knee stability can be achieved after all-inside reconstruction.

\section{Limitations}

This study had several constraints. First, the small sample size resulted in relatively low statistical power, and a study with a larger sample size is required to find more reliable conclusions. Second, data on clinical outcomes and SNQ values follow-up time were short, so the exact change tendency at 2-5 years remains unclear. Third, the SNQ values based on MRI mostly reflected the water content in the graft, which is still indirect evidence for graft maturity.

\section{Conclusion}

All-inside reconstruction achieves the same functional recovery as anatomical reconstruction but shows relatively insufficient stability, possibly due to prematurely returning to motion when the graft was immature. The graft maturity of all-inside reconstruction was the worst around the postoperative 6th month, worse than anatomical reconstruction, implying that rehabilitation strategies for all-inside reconstruction should be more conservative than those for anatomical reconstruction to achieve better stability. Additionally, there is no correlation between knee function and graft maturity, so rehabilitation strategy based solely on functional improvement should be made with caution. 


\section{Declarations}

Acknowledgements Thanks to all the researchers who contributed to the design, data collection and analysis, and writing of this article.

Authors' contributions (I) Conception and design: Rubing Lin, Qiuwen Zhong; (II) Administrative support: Changqing Jiang, Wei Li;(III) Provision of study materials or patients: Rong Huang, Qianhua Deng, Changqing Jiang, Wei Li; (IV) Collection and assembly of data:Rubing Lin, Qiuwen Zhong, Xiao Wu, Lei Cui; (V) Data analysis and interpretation: Rubing Lin, Qiuwen Zhong, Lei Cui;(VI) Manuscript writing: All authors; (VII) Final approval of manuscript: All authors.

Funding Shenzhen Health and Family Planning Commission funded 300,000 for this project.

Availability of data and materials The datasets used and/or analysed during the current study are available from the corresponding author on reasonable request. Declarations

Ethics approval and consent to participate The authors are accountable for all aspects of the work in ensuring that questions related to the accuracy or integrity of any part of the work are appropriately investigated and resolved. This retrospective study was carried out by the approval of institutional review/ethics committee of Peking University Shenzhen Hospital. This study was conducted in accordance with the Declaration of Helsinki (as revised in 2013). Informed consent was obtained by all participants and parent/legal guardian of minor participant (age less than 16 years) to participate in the study or to use their clinical and imaging data.

Consent for publication Not Applicable.

Competing interests All authors have completed the ICMJE uniform disclosure form. The authors declare that they have no competing interests.

Author details 1. Peking University Shenzhen Hospital, Clinical College of Anhui Medical University, Lianhua Road 1120, Futian District, Shenzhen City, Guangdong Province, P. R.China, 518036.2. Peking University Shenzhen Hospital, Lianhua Road 1120, FuTian District, ShenZhen City, GuangDong Province区 P.R.China, 518036.3. Huazhong University of Science and Technology Union Shenzhen Hospital, Taoyuan Road 89, Nanshan District, Shenzhen City, GuangDong Province区P.R.China, 518036.

\section{References}

1. Mall NA, Chalmers PN, Moric M, et al. Incidence and trends of anterior cruciate ligament reconstruction in the United States. Am J Sports Med 2014;42:2363-2370.

2. Liu S, Li H, Tao H, et al. A Randomized Clinical Trial to Evaluate Attached Hamstring Anterior Cruciate Ligament Graft Maturity With Magnetic Resonance Imaging. Am J Sports Med, 2018, 46(5):11431149. 
3. Poulsen E, Goncalves GH, Bricca A, et al. Knee osteoarthritis risk is increased 4-6 fold after knee injury - a systematic review and meta-analysis. Br J Sports Med, 2019, 53: 1454-1463.

4. Robson AM. Ruptured crucial ligaments and their repair by operation. Ann Surg,1903,37:716.

5. Lubowitz JH. No-tunnel anterior cruciate ligament reconstruction: the transtibial all-inside technique. Arthroscopy,2006,22(8): 1-11.

6. Lubowitz JH, Ahmad CS, Anderson K. All-inside anterior cruciate ligament graft-link technique: second-generation, no-incision anterior cruciate ligament reconstruction. Arthroscopy, 2011, 27(5): 717-727.

7. Lubowitz JH. All-inside ACL: retroconstruction controversies. Sports Med Arthrosc Rev,2010,18(1): 20-26.

8. Mayr R, Smekal V, Koidl C et al. ACL reconstruction with adjustable-length loop cortical button fixation results in less tibial tunnel widening compared with interference screw fixation. Knee Surg Sports Traumatol Arthrosc, 2020, 28: 1036-1044.

9. Desai VS, Anderson GR, Wu IT, et al. Anterior Cruciate Ligament Reconstruction With Hamstring Autograft: A Matched Cohort Comparison of the All- Inside and Complete Tibial Tunnel Techniques. Orthop J Sports Med, 2019, 7(1): 2325967118820297.

10. Lubowitz James H,Schwartzberg Randy,Smith Patrick,Randomized controlled trial comparing allinside anterior cruciate ligament reconstruction technique with anterior cruciate ligament reconstruction with a full tibial tunnel. Arthroscopy, 2013, 29: 1195-200.

11. Monaco E, Fabbri M, Redler A et al. Anterior cruciate ligament reconstruction is associated with greater tibial tunnel widening when using a bioabsorbable screw compared to an all-inside technique with suspensory fixation. Knee Surg Sports Traumatol Arthrosc, 2019, 27: 2577-2584.

12. Fu Chun-Wei,Chen Wei-Cheng,Lu Yung-Chang,Is all-inside with suspensory cortical button fixation a superior technique for anterior cruciate ligament reconstruction surgery? A systematic review and meta-analysis. BMC Musculoskelet Disord, 2020, 21: 445.

13. Bressy G, Brun V, Ferrier A, et al.Lack of stability at more than 12 months of follow-up after anterior cruciate ligament reconstruction using all-inside quadruple-stranded semitendinosus graft with adjustable cortical button fixation in both femoral and tibial sides. Orthop Traumatol Surg Res,2016,102(7).

14. Fu Chun-Wei, Chen Wei-Cheng, Lu Yung-Chang, Is all-inside with suspensory cortical button fixation a superior technique for anterior cruciate ligament reconstruction surgery? A systematic review and meta-analysis.[J].BMC Musculoskelet Disord, 2020, 21: 445.

15. Connaughton AJ, Geeslin AG, Uggen CW. All-inside ACL reconstruction: How does it compare to standard ACL reconstruction techniques? J Orthop, 2017,14(2): 241-246.

16. Ma Yong,Murawski Christopher D,Rahnemai-Azar Amir Ata et al. Graft maturity of the reconstructed anterior cruciate ligament 6 months postoperatively: a magnetic resonance imaging evaluation of quadriceps tendon with bone block and hamstring tendon autografts.[J].Knee Surg Sports Traumatol Arthrosc, 2015, 23: 661-8. 
17. Van Dyck Pieter,Zazulia Katja,Smekens Céline et al. Assessment of Anterior Cruciate Ligament Graft Maturity With Conventional Magnetic Resonance Imaging: A Systematic Literature Review.[J].Orthop J Sports Med, 2019, 7: 2325967119849012.

18. Chen $L, W u Y$, Lin $G$, et al. Graft bending angle affects allograft tendon maturity early after anterior cruciate ligament reconstruction. Knee Surg Sports Traumatol Arthrosc, 2018, 26(10):3048-3054.

19. Pauzenberger $L$, Schurz M. "Ligamentization" in hamstring tendongrafts after anterior cruciate ligament reconstruction: a systematic review of the literature and a glimpse into the future. Arthroscopy the Journal of Arthroscopic \& Related Surgery, 2013, 29(10):1721.

20. Li Q, Zhang Y, Zhan L, et al. Correlation Analysis of Magnetic Resonance Imaging-Based Graft Maturity and Outcomes After Anterior Cruciate Ligament Reconstruction Using International Knee Documentation Committee Score. American Journal of Physical Medicine \& Rehabilitation, 2019, 98(5):387-391.

21. Scheffler SU, Unterhauser FN, Weiler A. Graft remodeling and ligamentization after cruciate ligament reconstruction. Knee Surg Sports Traumatol Arthrosc, 2008, 16(9):834-842.

22. Amiel D, Kleiner JB, Roux RD, et al. The phenomenon of "ligamentization": anterior cruciate ligament reconstruction with autogenous patellar tendon. Journal of Orthopaedic Research, 2010, 4(2):162172.

23. Janssen RP, Scheffler SU. Intra-articular remodelling of hamstring tendon grafts after anterior cruciate ligament reconstruction. Knee Surg Sports Traumatol Arthrosc, 2014, 22(9):2102-2108.

24. Kim BH, Kim JI, Lee O, et al. Preservation of remnant with poor synovial coverage has no beneficial effect over remnant sacrifice in anterior cruciate ligament reconstruction. Knee Surg Sports Traumatol Arthrosc, 2018, 26(8):2345-2352.

25. Nakayama H, Kambara S, Iseki T, et al. Double-bundle anterior cruciate ligament reconstruction with and without remnant preservation-Comparison of early postoperative outcomes and complications. Knee, 2017, 24(5):1039-1046.

26. Liu S, Li H, Tao H, et al. A Randomized Clinical Trial to Evaluate Attached Hamstring Anterior Cruciate Ligament Graft Maturity With Magnetic Resonance Imaging. Am J Sports Med, 2018, 46(5):11431149.

27. Ruffilli A, Pagliazzi G, Ferranti E, et al. Hamstring graft tibial insertion preservation versus detachment in anterior cruciate ligament reconstruction: a prospective randomized comparative study. Eur $\mathrm{J}$ Orthop Surg Traumatol, 2016, 26(6):657-664.

28. Chen Linhai,Wu Yibing,Lin Guanghao et al. Graft bending angle affects allograft graft maturity early after anterior cruciate ligament reconstruction. Knee Surg Sports Traumatol Arthrosc, 2018, 26: 30483054.

29. Lind M,Feller J,Webster KE.Bone tunnel widening after anterior cruciate ligament reconstruction using EndoButton or EndoButton continuous loop.Arthroscopy, 2009, 25(11):1275-1280.

30. de Sa D, Shanmugaraj A, Weidman M et al. All-Inside Anterior Cruciate Ligament Reconstruction-A Systematic Review of Techniques, Outcomes, and Complications. J Knee Surg, 2018, 31: 895-904. 
31. Hofbauer Marcus,Soldati Francesco,Szomolanyi Pavol et al. Hamstring tendon autografts do not show complete graft maturity 6 months postoperatively after anterior cruciate ligament reconstruction. Knee Surg Sports Traumatol Arthrosc, 2019, 27: 130-136.

32. Zhang Yuhan,Liu Shaohua,Chen Qingyan et al. Maturity Progression of the Entire Anterior Cruciate Ligament Graft of Insertion-Preserved Hamstring Tendons by 5 Years: A Prospective Randomized Controlled Study Based on Magnetic Resonance Imaging Evaluation. Am J Sports Med, 2020, 48 : 2970-2977.

\section{Figures}




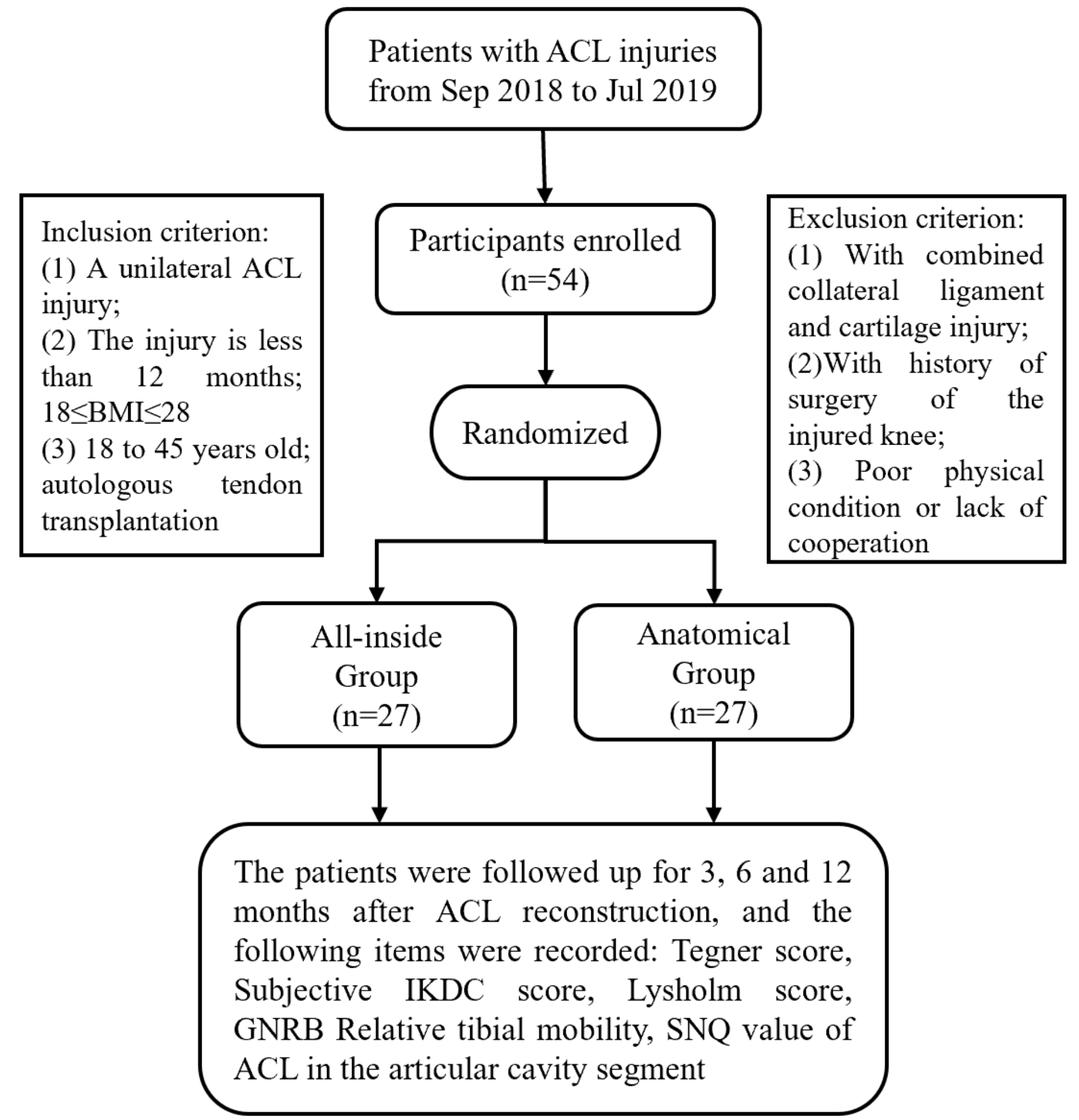

Figure 1

Flow diagram of the whole study. 


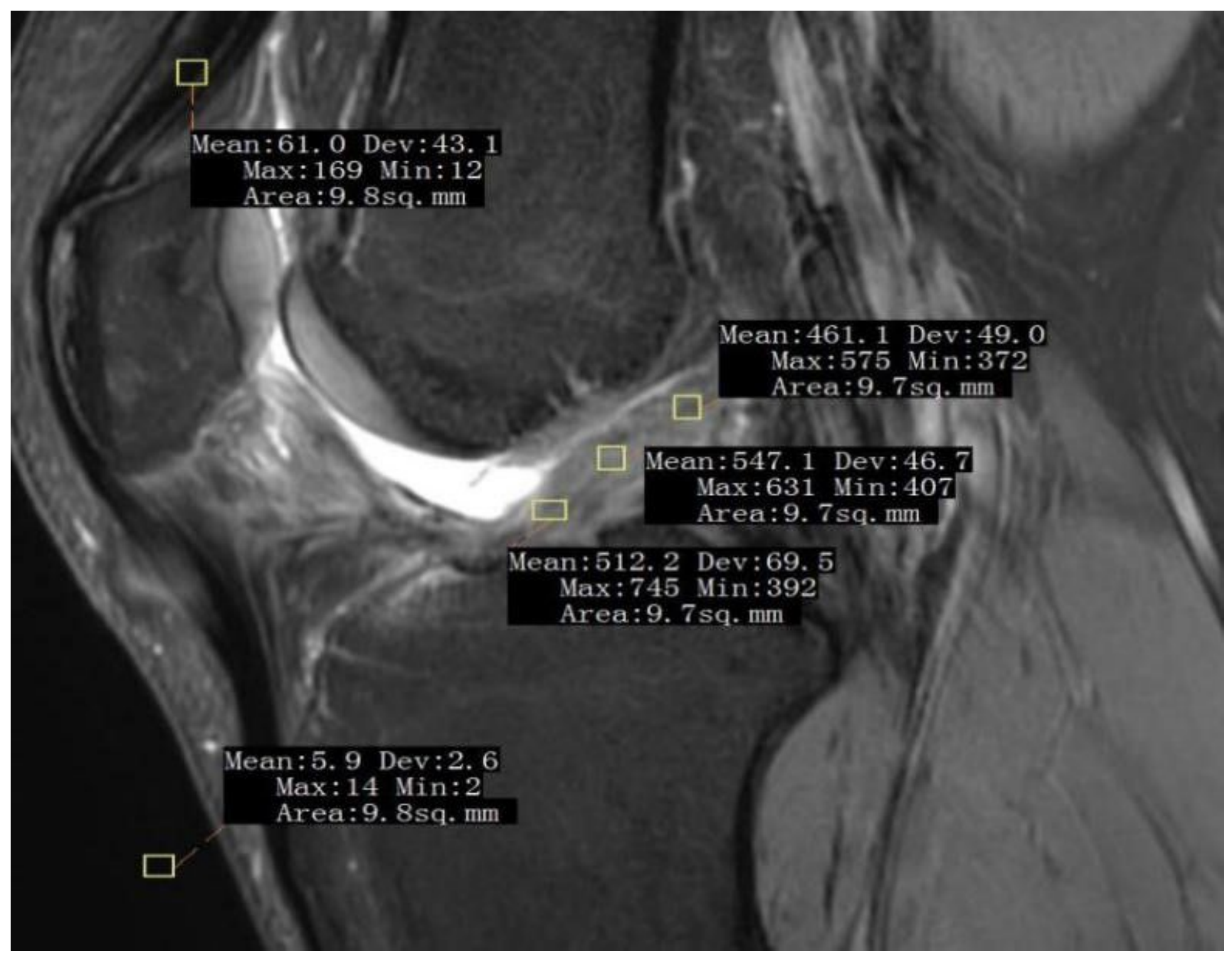

\section{Figure 2}

The location of the proximal, distal, and middle regions of interest (ROI) for intra-articular graft, and the location of the quadriceps tendon and background ROI.

展

\section{Figure 3}

Tendon graft preparation method and drilling of tunnel "bone socket" structure of all-inside single-bundle anterior cruciate ligament reconstruction technique. 


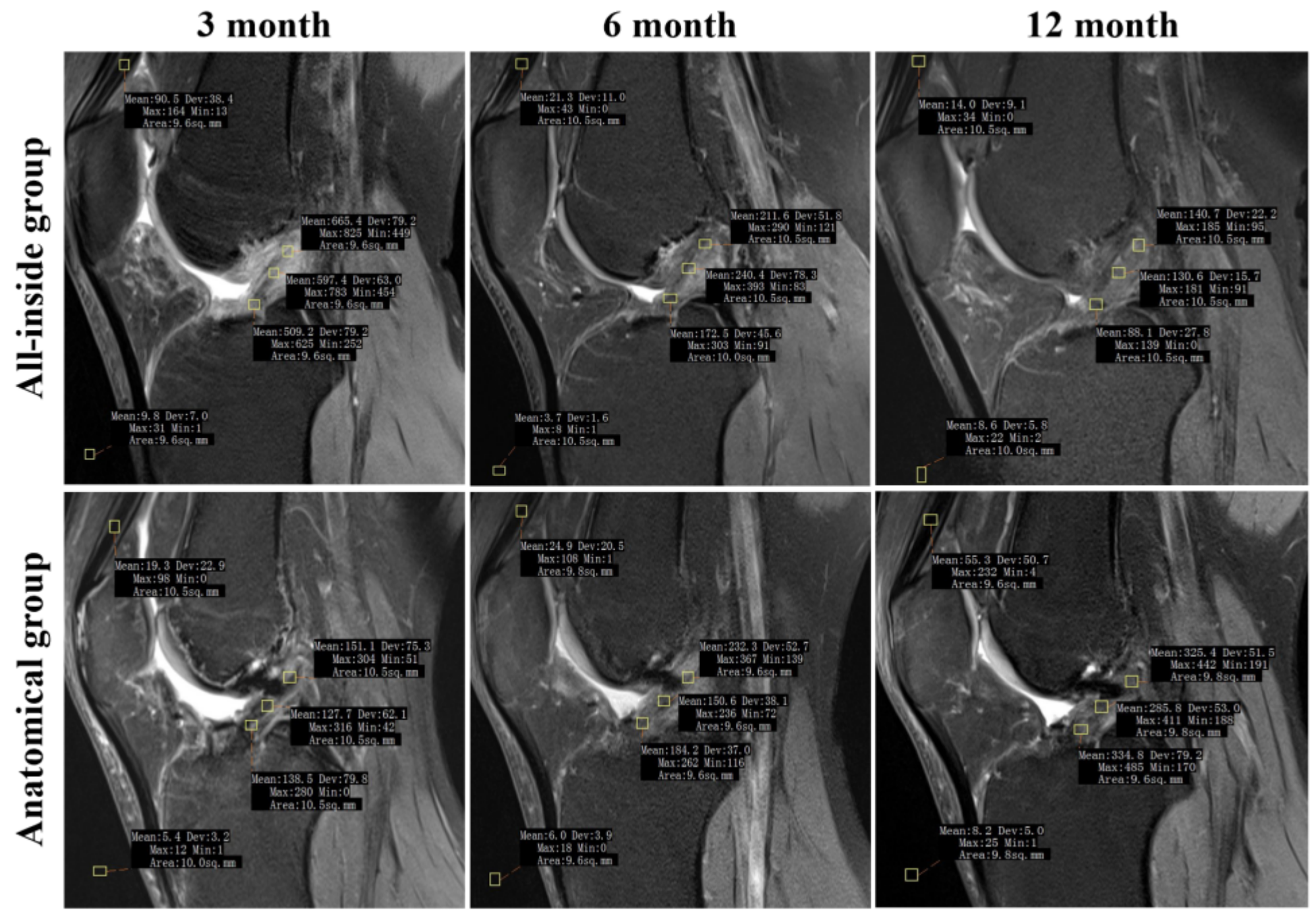

Figure 4

MRI appearance and signal value measurement of intra-articular graft in the same patient during the 3rd, 6 th, and 12th months after surgery. 


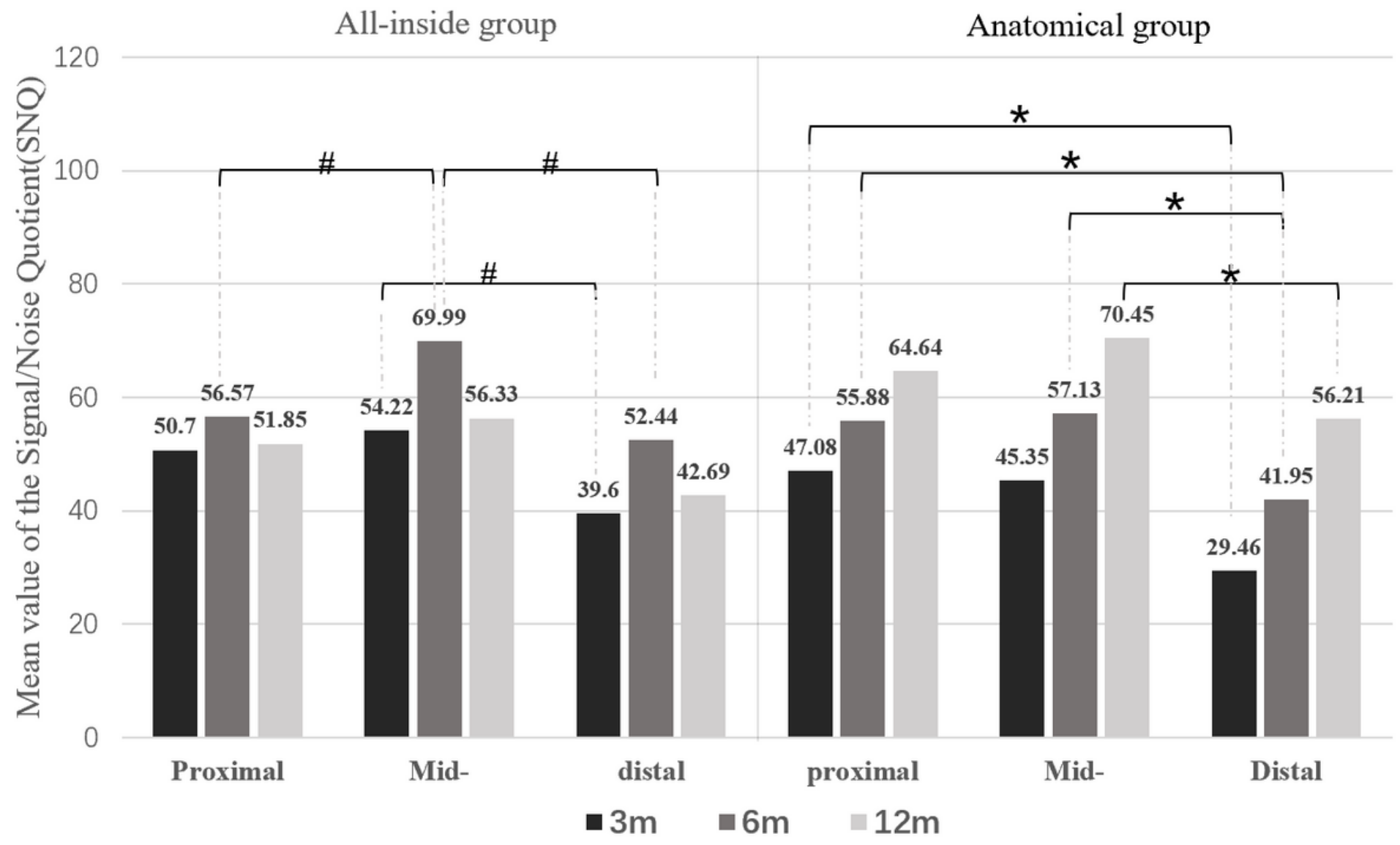

Figure 5

The SNQ of the proximal, distal, and middle ROI of the all-inside and anatomical group during postoperative 3rd, 6th, and 12th month. All-inside group graft SNQ presented a "mountain" shape, and the anatomical group graft SNQ presented a "staircase" shape. Both groups showed the highest SNQ of the graft in the middle region, followed by the proximal region, and the lowest was the distal region. "\#" and " $\star$ " indicated a statistical difference between the two. 


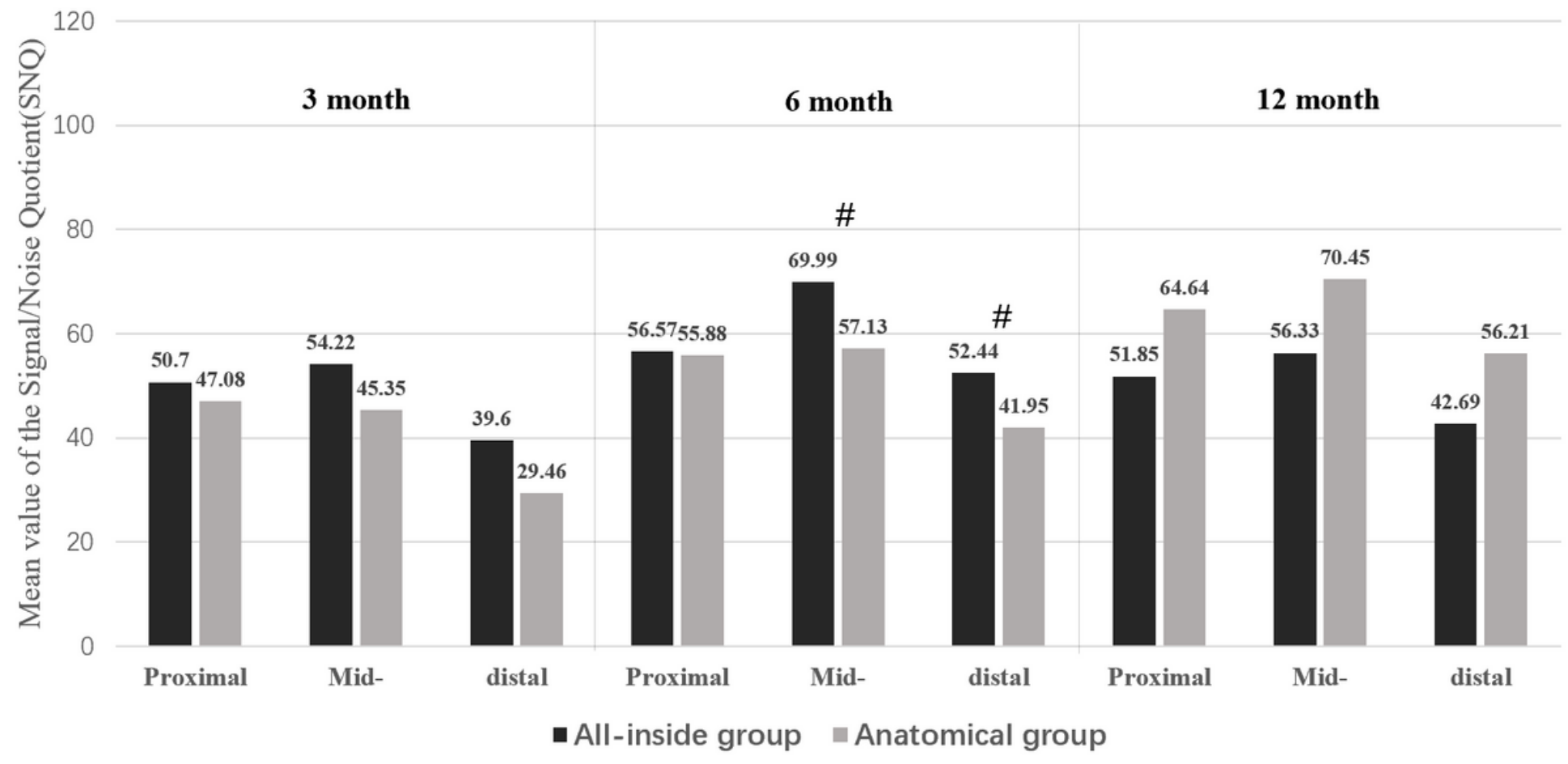

Figure 6

The SNQ of the proximal, distal, and middle ROI of the all-inside and anatomical group at postoperative $3 \mathrm{rd}, 6$ th, and 12th month. The SNQ of the all-inside group in the middle and distal region was significantly

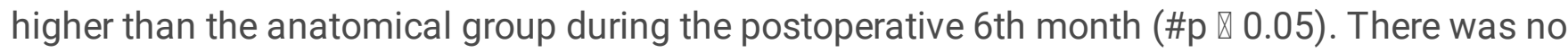
statistical difference between the rest of the time or region $(p \otimes 0.05)$. 